\title{
ON ANNIHILATOR IDEALS OF SKEW MONOID RINGS*
}

\author{
LIU ZHONGKUI and YANG XIAOYAN \\ Department of Mathematics, Northwest Normal University \\ Lanzhou 730070, Gansu, People's Republic of China \\ e-mail:liuzk@nwnu.edu.cn
}

(Received 31 March 2008; revised 10 January 2009; accepted 25 August 2009)

\begin{abstract}
A ring $R$ is called a left APP-ring if the left annihilator $l_{R}(R a)$ is pure as a left ideal of $R$ for every $a \in R ; R$ is called (left principally) quasi-Baer if the left annihilator of every (principal) left ideal of $R$ is generated by an idempotent. Let $R$ be a ring and $M$ an ordered monoid. Assume that there is a monoid homomorphism $\phi: M \longrightarrow A u t(R)$. We give a necessary and sufficient condition for the skew monoid ring $R * M$ (induced by $\phi$ ) to be left APP (left principally quasi-Baer, quasi-Baer, respectively).
\end{abstract}

2000 Mathematics Subject Classification. 16S35, 16S36.

1. Introduction. Throughout this paper, $R$ denotes a ring with unity. Recall that $R$ is a right PP-ring if the right annihilator of an element of $R$ is generated by an idempotent. Armendariz showed that polynomial rings over right PP-rings need not be right PP in the example in [1]. Also the concept of right PP-rings is not a Morita invariant property because $\mathbb{Z}[x]$ is PP but the $2 \times 2$ full matrix ring over $\mathbb{Z}[x]$ is not a right PP-ring [1]. In order to consider the natural question of how much of the right $\mathrm{PP}$ condition transfers to polynomial rings or matrix rings, a concept of left APP-rings was introduced and considered in [15]. By [15], Proposition 2.3, right PP-rings are left APP. It was shown in [15], Theorem 3.8 and Corollary 3.12, that the left APP condition is a Morita invariant property and transfers to a variety of polynomial extensions.

On the other hand, a ring $R$ is (quasi-)Baer if the left annihilator of every nonempty subset (every left ideal) of $R$ is generated by an idempotent of $R$. Clark defined quasi-Baer rings in [7] and used them to characterise when a finite dimensional algebra with unity over an algebraically closed field is isomorphic to a twisted matrix units semi-group algebra. As a generalisation of quasi-Baer rings, Birkenmeier, Kim and Park in [5] introduced the concept of left principally quasi-Baer rings. A ring $R$ is called left principally quasi-Baer if the left annihilator of a principal left ideal of $R$ is generated by an idempotent. Observe that biregular rings and quasi-Baer rings are left principally quasi-Baer. Clearly the concept of left APP-rings is a common generalisation of left principally quasi-Baer rings and right PP-rings. For more details and examples of quasi-Baer rings, left principally quasi-Baer rings and left APP-rings, see $[2-6,12,13$ and 15].

In this paper we consider the left APP property, left principal quasi-Baerness and quasi-Baerness of the skew monoid ring $R * M$. Some necessary and sufficient

\footnotetext{
* Supported by National Natural Science Foundation of China (10961021), TRAPOYT and the Cultivation Fund of the Key Scientific and Technical Innovation Project, Ministry of Education of China.
} 
conditions for the skew monoid ring $R * M$ to be left APP (left principally quasi-Baer, quasi-Baer) are obtained.

Let $R$ be a ring, and let $M$ be an ordered monoid. Assume that there exists a monoid homomorphism $\phi: M \longrightarrow \operatorname{Aut}(R)$. For any $g \in M$ and any $r \in R$, we denote by $r^{g}$ the image of $r$ under $\phi(g)$. Then we can form a skew monoid ring $R * M$ (induced by the monoid homomorphism $\phi$ ) by taking its elements to be finite formal combinations $\sum_{g \in M} a_{g} g$, with multiplication induced by

$$
\left(a_{g} g\right)\left(b_{h} h\right)=\left(a_{g} b_{h}^{g}\right)(g h)
$$

If $\phi$ is weakly rigid (that is to say $a b=0$ implies $a^{g} b=a b^{g}=0$ for any $a, b \in R$ and any $g \in M)$, then it has been proved in [14] that the skew monoid ring $R * M$ is quasi-Baer if and only if $R$ is quasi-Baer. If $R$ is $\phi$-compatible, then it has been proved in [8] that $R * M$ is (left principally) quasi-Baer if and only if $R$ is (left principally) quasi-Baer. It was shown in [15], Theorem 3.10, that if $R$ is a left APP-ring, $M$ is a u.p.-monoid, and the monoid homomorphism $\phi: M \longrightarrow \operatorname{Aut}(R)$ satisfies the condition that for every $a \in R$, the left ideal $\sum_{g \in M} R a^{g}$ is finitely generated, then the skew monoid ring $R * M$ (induced by the monoid homomorphism $\phi$ ) is a left APP-ring. When $M$ is a group or $\operatorname{Im}(\phi)$ is a group, a necessary and sufficient condition for the skew monoid ring $R * M$ to be (left principally) quasi-Baer was given in [9]. In this paper we will show that for an ordered monoid $M$ and a monoid homomorphism $\phi: M \longrightarrow \operatorname{Aut}(R)$, the skew monoid ring $R * M$ is a left APP-ring (a left principally quasi-Baer ring, a quasi-Baer ring, respectively) if and only if the left annihilator $l_{R}\left(\sum_{g \in M} R a^{g}\right)$ is right s-unital for every $a \in R$. (The left annihilator of $\sum_{g \in M} R a^{g}$ is generated by an idempotent for every $a \in R$, and the left annihilator of left ideal $\sum_{b \in S} \sum_{g \in M} R b^{g}$ of $R$ is generated by an idempotent for every subset $S$ of $R$, respectively.)

For a non-empty subset $Y$ of $R, l_{R}(Y)$ and $r_{R}(Y)$ denote the left and right annihilators of $Y$ in $R$, respectively. A monoid $M$ is said to be ordered if the elements of $M$ are linearly ordered with respect to the relation $<$ and if for all $x, y, z \in M, x<y$ implies $z x<z y$ and $x z<y z$. It is well known that any submonoid of a free group or a torsion-free nilpotent group is an ordered monoid. We denote by $\eta$ the identity of a monoid $M$.

2. Left APP-rings. An ideal $I$ of $R$ is said to be right s-unital if for each $a \in I$ there exists an element $x \in I$ such that $a x=a$. Note that if $I$ and $J$ are right s-unital ideals, then so is $I \cap J$. (If $a \in I \cap J$, then $a \in a I J \subseteq a(I \cap J)$.) It follows from [17], Theorem 1, that $I$ is right s-unital if and only if for any finitely many elements $a_{1}, a_{2}, \ldots, a_{n} \in I$ there exists an element $x \in I$ such that $a_{i}=a_{i} x, i=1,2, \ldots, n$. A submodule $N$ of a left $R$-module $M$ is called a pure submodule if $L \otimes_{R} N \longrightarrow L \otimes_{R} M$ is a monomorphism for every right $R$-module $L$. By [16], Proposition 11.3.13, an ideal $I$ is right s-unital if and only if $R / I$ is flat as a left $R$-module if and only if $I$ is pure as a left ideal of $R$.

By [15], a ring $R$ is called a left APP-ring if the left annihilator $l_{R}(R a)$ is right s-unital as an ideal of $R$ for any element $a \in R$.

Right APP-rings may be defined analogously. Clearly every left principally quasi-Baer ring is a left APP-ring. (Thus the class of left APP-rings includes all biregular rings and all quasi-Baer rings.) From [15], it follows that right PPrings are left APP and left APP-rings are quasi-Armendariz in the sense that 
whenever $f(x)=a_{0}+a_{1} x+\cdots+a_{m} x^{m}, g(x)=b_{0}+b_{1} x+\cdots+b_{n} x^{n} \in R[x]$ satisfy $f(x) R[x] g(x)=0$, we have $a_{i} R b_{j}=0$ for each $i$ and $j$ (see for example [10]).

Let $M$ be a monoid and $\phi: M \longrightarrow \operatorname{Aut}(R)$ a monoid homomorphism. The ring $R$ is called left $M$-APP if the left annihilator $l_{R}\left(\sum_{g \in M} R b^{g}\right)$ is right s-unital for every $b \in R$. Clearly if $\phi(g)=1$ for every $g \in M$, then $R$ is left $M$-APP if and only if $R$ is left APP. It is easy to see that if $R$ is a left Noetherian and left APP-ring, then $R$ is left $M$-APP for any monoid $M$ (in fact, there exists a maximal element $\sum_{g \in N_{0}} R b^{g}$ in the set $\left\{\sum_{g \in N} R b^{g}|N \subseteq M| N \mid,<\infty\right\}$, which is unique, and so $l_{R}\left(\sum_{g \in M} R b^{g}\right)=$ $l_{R}\left(\sum_{g \in N_{0}} R b^{g}\right)=\cap_{g \in N_{0}} l_{R}\left(R b^{g}\right)$ is right s-unital $)$.

REMARK 1. (1) It follows from [15], Theorem 3.10, that if $R$ is a left APP-ring and $M$ an ordered monoid and if the monoid homomorphism $\phi: M \longrightarrow \operatorname{Aut}(R)$ satisfies the condition that for every $a \in R$, the left ideal $\sum_{g \in M} R a^{g}$ is finitely generated, then the skew monoid ring $R * M$ (induced by the monoid homomorphism $\phi$ ) is a left APP-ring. Thus, by Theorem 2, $R$ is a left $M$-APP-ring. Remark 3.11 of [15] gave some examples of left $M$-APP-rings.

(2) For a given left APP-ring $T$, let

$$
R=\left\{\left(a_{n}\right)_{n \in \mathbb{Z}} \in \prod T \mid a_{n} \text { is eventually constant }\right\}
$$

which is a subring of the countably infinite direct product $\prod_{\mathbb{Z}} T$. Define an automorphism $\sigma$ of $R$ by $\sigma\left(a_{n}\right)_{n \in \mathbb{Z}}=\left(a_{n+1}\right)_{n \in \mathbb{Z}}$. Let $M=\mathbb{N} \cup\{0\}$. Define $\phi: M \longrightarrow \operatorname{Aut}(R)$ via $\phi(0)=1$ and $\phi(n)=\sigma^{n}$ for every $n \in \mathbb{N}$. Suppose that $w=\left(\ldots, a, a, a_{s}, a_{s+1}, \ldots, a_{t}, a, a, \ldots\right) \in l_{R}\left(\sum_{g \in M} R b^{g}\right)$, where $b=\left(b_{n}\right)_{n \in \mathbb{Z}} \in R$. Then $w R b^{g}=0$ for each $g \in M$. Thus for any $s \leq n \leq t, a_{n} T b_{n}=0, a_{n} T b_{n-1}=0, a_{n} T b_{n-2}=$ $0, \ldots$ Since $\left(b_{n}\right)_{n \in \mathbb{Z}}$ is eventually constant, the left ideal $T b_{n}+T b_{n-1}+\cdots$ is finitely generated. By Proposition 2.6 of $[\mathbf{1 5}], l_{T}\left(T b_{n}+T b_{n-1}+\cdots\right)$ is right sunital. Thus $a_{n}=a_{n} a_{n}^{\prime}$ for some $a_{n}^{\prime} \in l_{T}\left(T b_{n}+T b_{n-1}+\cdots\right)$. Similarly $a=a a^{\prime}$ for some $a^{\prime} \in l_{T}\left(\sum_{n \in \mathbb{Z}} T b_{n}\right)$. Now it is easy to see that $w=w w^{\prime}$, where $w^{\prime}=$ $\left(\ldots, a^{\prime}, a^{\prime}, a_{s}^{\prime}, \ldots, a_{t}^{\prime}, a^{\prime}, a^{\prime}, \ldots\right) \in l_{R}\left(\sum_{g \in M} R b^{g}\right)$. Therefore $R$ is left $M$-APP.

If we take $T=S[[x]]$, where

$$
S=\left(\prod_{i=1}^{\infty} \mathbb{Z} / 2 \mathbb{Z}\right) /\left(\bigoplus_{i=1}^{\infty} \mathbb{Z} / 2 \mathbb{Z}\right),
$$

then, by Example 2.5 of [15], $T$ is an APP-ring, but $T$ is neither PP nor principally quasi-Baer. Thus $R$ is neither PP nor principally quasi-Baer.

(3) The following example (see [9]) shows that left $M$-APP rings need not be left APP. Let $F$ be a field; let $A=F[s, t]$ be a commutative polynomial ring; and consider the ring $R=A /(s t)$. Let $\bar{s}=s+(s t)$ and $\bar{t}=t+(s t)$ in $R$. Define an automorphism $\sigma$ of $R$ by $\sigma(\bar{s})=\bar{t}$ and $\sigma(\bar{t})=\bar{s}$. Then $l_{R}(R \bar{s})=R \bar{t}$. Clearly this ideal is not right sunital. Thus $R$ is not a left APP-ring. By Example 2 of [9], any non-zero ideal $I$ of $R$ with $\sigma(I)=I$ is essential in $R$, and so $l_{R}(I)=0$. (Note that $R$ is a reduced ring.) Let $M=\mathbb{N} \cup\{0\}$. Define $\phi: M \longrightarrow \operatorname{Aut}(R)$ via $\phi(0)=1$ and $\phi(n)=\sigma^{n}$ for every $n \in \mathbb{N}$. Therefore $R$ is $M$-APP.

The following is our main result which gives a necessary and sufficient condition for the skew monoid ring $R * M$ to be a left APP-ring. 
THEOREM 2. Let $R$ be a ring, $M$ an ordered monoid and $\phi: M \longrightarrow A u t(R)$ a monoid homomorphism. Then the following are equivalent:

(1) The skew monoid ring $R * M$ is a left APP-ring.

(2) $R$ is a left $M$-APP-ring.

Proof. (2) $\Longrightarrow(1)$. Let $\alpha=a_{1} g_{1}+a_{2} g_{2}+\cdots+a_{n} g_{n}$, and let $\beta=b_{1} h_{1}+b_{2} h_{2}$ $+\cdots+b_{m} h_{m} \in R * M$ satisfy $\alpha(R * M) \beta=0$. Without loss of generality, we assume that $g_{i}<g_{j}$ and $h_{i}<h_{j}$ if $i<j$. Suppose that $c_{1}, c_{2}, \ldots, c_{n} \in R$ are such that $a_{i}=c_{i}^{g_{i}}$ for $i=1,2, \ldots, n$. We will show that $c_{i} \in l_{R}\left(\sum_{g \in M} R b_{j}^{g}\right)$ for $i=1,2, \ldots, n, j=1,2, \ldots, m$ by induction on $n$.

For any $c \in R$ and any $g \in M$, from $\alpha(R * M) \beta=0$ it follows that

$$
\left(a_{1} g_{1}+a_{2} g_{2}+\cdots+a_{n} g_{n}\right)(c g)\left(b_{1} h_{1}+b_{2} h_{2}+\cdots+b_{m} h_{m}\right)=0 .
$$

Considering the coefficient of the largest element $g_{n} g h_{m}$ in above, we obtain $a_{n} c^{g_{n}} b_{m}^{g_{n} g}=$ 0 . This implies that $\left(c_{n} c b_{m}^{g}\right)^{g_{n}}=0$. Thus $c_{n} c b_{m}^{g}=0$, since $(-)^{g_{n}}$ is an automorphism of $R$. So $c_{n} R b_{m}^{g}=0$ for all $g \in M$, which implies that $c_{n}\left(\sum_{g \in M} R b_{m}^{g}\right)=0$. That is to say $c_{n} \in l_{R}\left(\sum_{g \in M} R b_{m}^{g}\right)$. By (2), $l_{R}\left(\sum_{g \in M} R b_{m}^{g}\right)$ is right s-unital. Thus there exists $e \in l_{R}\left(\sum_{g \in M} R b_{m}^{g}\right)$ such that $c_{n}=c_{n} e$. Now for every $c \in R$ and every $g \in M$, we have

$$
\begin{aligned}
0= & \left(a_{1} g_{1}+a_{2} g_{2}+\cdots+a_{n} g_{n}\right)(e c g)\left(b_{1} h_{1}+b_{2} h_{2}+\cdots+b_{m} h_{m}\right) \\
= & a_{1} e^{g_{1}} c^{g_{1}} b_{1}^{g_{1} g} g_{1} g h_{1}+\cdots+a_{n-1} e^{g_{n-1}} c^{g_{n-1}} b_{m}^{g_{n-1} g} g_{n-1} g h_{m} \\
& +a_{n} e^{g_{n}} c^{g_{n}} b_{m-1}^{g_{n} g} g_{n} g h_{m-1}+a_{n} e^{g_{n}} c^{g_{n}} b_{m}^{g_{n} g} g_{n} g h_{m} .
\end{aligned}
$$

Since $a_{n} e^{g_{n}} c^{g_{n}} b_{m}^{g_{n} g}=a_{n}\left(e c b_{m}^{g}\right)^{g_{n}}=0, a_{n-1} e^{g_{n-1}} c^{g_{n-1}} b_{m}^{g_{n-1} g}=a_{n-1}\left(e c b_{m}^{g}\right)^{g_{n-1}}=0$, considering the coefficient of the largest element $g_{n} g h_{m-1}$ in above, we have $a_{n} e^{g_{n}} c^{g_{n}} b_{m-1}^{g_{n} g}=0$. Thus

$$
\left(c_{n} c b_{m-1}^{g}\right)^{g_{n}}=\left(c_{n} e c b_{m-1}^{g}\right)^{g_{n}}=c_{n}^{g_{n}} e^{g_{n}} c^{g_{n}} b_{m-1}^{g_{n} g}=a_{n} e^{g_{n}} c^{g_{n}} b_{m-1}^{g_{n} g}=0,
$$

which implies that $c_{n} R b_{m-1}^{g}=0$ for every $g \in M$. Hence $c_{n} \in l_{R}\left(\sum_{g \in M} R b_{m}^{g}\right) \cap$ $l_{R}\left(\sum_{g \in M} R b_{m-1}^{g}\right)$. Noting that $l_{R}\left(\sum_{g \in M} R b_{m}^{g}\right)$ and $l_{R}\left(\sum_{g \in M} R b_{m-1}^{g}\right)$ are right sunital ideals of $R$, so is $l_{R}\left(\sum_{g \in M} R b_{m}^{g}\right) \cap l_{R}\left(\sum_{g \in M} R b_{m-1}^{g}\right)$. Thus there exists $f \in l_{R}\left(\sum_{g \in M} R b_{m}^{g}\right) \cap l_{R}\left(\sum_{g \in M} R b_{m-1}^{g}\right)$ such that $c_{n}=c_{n} f$. Now for any $g \in M$ and any $c \in R$ we have $\left(a_{1} g_{1}+a_{2} g_{2}+\cdots+a_{n} g_{n}\right)(f c g)\left(b_{1} h_{1}+b_{2} h_{2}+\cdots+b_{m} h_{m}\right)=0$. Continuing this process, we obtain

$$
c_{n} \in \cap_{j=1}^{m} l_{R}\left(\sum_{g \in M} R b_{j}^{g}\right) .
$$

Thus for any $c \in R$ and any $g \in M,\left(a_{n} g_{n}\right)(c g)\left(b_{1} h_{1}+b_{2} h_{2}+\cdots+b_{m} h_{m}\right)=$ $a_{n} c^{g_{n}} b_{1}^{g_{n} g} g_{n} g h_{1}+\cdots+a_{n} c^{g_{n}} b_{m}^{g_{n} g} g_{n} g h_{m}=\left(c_{n} c b_{1}^{g}\right)^{g_{n}} g_{n} g h_{1}+\cdots+\left(c_{n} c b_{m}^{g}\right)^{g_{n}} g_{n} g h_{m}=0$.

So we have

$$
\left(a_{1} g_{1}+a_{2} g_{2}+\cdots+a_{n-1} g_{n-1}\right)(R * M)\left(b_{1} h_{1}+b_{2} h_{2}+\cdots+b_{m} h_{m}\right)=0 .
$$

Now using induction on $n$, we obtain that $c_{i} \in \cap_{j=1}^{m} l_{R}\left(\sum_{g \in M} R b_{j}^{g}\right)$ for all $i$. Since $l_{R}\left(\sum_{g \in M} R b_{1}^{g}\right), \ldots, l_{R}\left(\sum_{g \in M} R b_{m}^{g}\right)$ are right s-unital ideals, it is clear that 
$\cap_{j=1}^{m} l_{R}\left(\sum_{g \in M} R b_{j}^{g}\right)$ is right s-unital. Thus there exists $e \in \cap_{j=1}^{m} l_{R}\left(\sum_{g \in M} R b_{j}^{g}\right)$ such that $c_{i}=c_{i} e, i=1,2, \ldots, n$. Now we have

$$
\begin{aligned}
\alpha(e \eta) & =\left(a_{1} g_{1}+a_{2} g_{2}+\cdots+a_{n} g_{n}\right)(e \eta) \\
& =\sum_{i=1}^{n} a_{i} e^{g_{i}} g_{i}=\sum_{i=1}^{n}\left(c_{i} e\right)^{g_{i}} g_{i}=\sum_{i=1}^{n} c_{i}^{g_{i}} g_{i}=\sum_{i=1}^{n} a_{i} g_{i} \\
& =\alpha .
\end{aligned}
$$

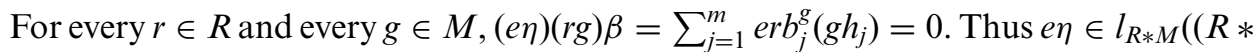
$M) \beta$ ). This shows that $R * M$ is a left APP-ring.

$(1) \Longrightarrow(2)$. Suppose that $R * M$ is a left APP-ring. Let $b \in R$ and $a \in l_{R}\left(\sum_{g \in M} R b^{g}\right)$. Then $(a \eta)(R * M)(b \eta)=0$. Thus there exists $c_{0} \eta+c_{1} g_{1}+\cdots+c_{n} g_{n} \in R * M$ such that $a \eta=(a \eta)\left(c_{0} \eta+c_{1} g_{1}+\cdots+c_{n} g_{n}\right)$ and $\left(c_{0} \eta+c_{1} g_{1}+\cdots+c_{n} g_{n}\right)(R * M)(b \eta)=0$, where $c_{i} \in R$ and $\eta, g_{1}, \ldots, g_{n}$ are distinct elements of $M$. It is easy to see that $a=a c_{0}$. Note that $M$ is cancellative. For any $r \in R$ and any $g \in M$, from $\left(c_{0} \eta+\right.$ $\left.c_{1} g_{1}+\cdots+c_{n} g_{n}\right)(r g)(b \eta)=0$ it follows that $c_{0} r b^{g} g=0$, which implies that $c_{0} r b^{g}=0$. Thus $c_{0}\left(\sum_{g \in M} R b^{g}\right)=0$. This shows that $R$ is a left $M$-APP-ring.

COROLlary 3. Let $R$ be a ring and $M$ an ordered monoid. Then the monoid ring $R[M]$ is left $A P P$ if and only if $R$ is left $A P P$.

COROLlaRY 4. Let $R$ be a ring and $\sigma$ a ring automorphism of $R$. Then the ring $R[x ; \sigma]$ (respectively $R\left[x, x^{-1} ; \sigma\right]$ ) is left $A P P$ if and only if the left annihilator of $\sum_{i=0}^{\infty} R \sigma^{i}(b)$ (respectively $\sum_{i=-\infty}^{\infty} R \sigma^{i}(b)$ ) is right s-unital for every $b \in R$.

Proof. Define a homomorphism $\phi: \mathbb{N} \cup\{0\} \longrightarrow \operatorname{Aut}(R)(\phi: \mathbb{Z} \longrightarrow \operatorname{Aut}(R))$ of monoids via $\phi(i)=\sigma^{i}$. Then the result follows from Theorem 2 .

3. Left principally quasi-Baer rings and quasi-Baer rings. Let $R$ be a ring, $M$ a monoid and $\phi: M \longrightarrow \operatorname{Aut}(R)$ a monoid homomorphism; $R$ is called a left $M$ principally quasi-Baer ring if for any $a \in R$, the left annihilator of $\sum_{g \in M} R a^{g}$ is generated by an idempotent. For the condition that $M$ is a group, left $M$-principally quasi-Baer rings were considered by Y. Hirano in [9]. Note that by Remark 1(3), left $M$-principally quasi-Baer rings need not be left principally quasi-Baer.

There are a lot of results concerning quasi-Baerness and left principal quasiBaerness of polynomial extensions of a ring. G. F. Birkenmeier, J. Y. Kim and J. K. Park showed in [4], Theorem 1.8, that $R$ is quasi-Baer if and only if $R[X]$ is quasi-Baer if and only if $R[[X]]$ is quasi-Baer if and only if $R\left[x, x^{-1}\right]$ is quasi-Baer if and only if $R\left[\left[x, x^{-1}\right]\right]$ is quasi-Baer, where $X$ is an arbitrary non-empty set of not necessarily commuting indeterminates. Furthermore, it was shown in [4], Theorem 1.2, that if $R$ is quasi-Baer, then so are $R[x ; \sigma], R[[x ; \sigma]], R\left[x, x^{-1} ; \sigma\right]$ and $R\left[\left[x, x^{-1} ; \sigma\right]\right]$. It was proved in [3], Theorem 2.1, that a ring $R$ is left principally quasi-Baer if and only if $R[x]$ is left principally quasi-Baer. C. Y. Hong, N. K. Kim and T. K. Kwak showed in [11], Corollaries 12, 15 and 22, that if $\sigma$ is a rigid endomorphism of $R$, then $R$ is a quasi-Baer (respectively left principally quasi-Baer) ring if and only if $R[x ; \sigma, \delta]$ is a quasi-Baer (respectively left principally quasi-Baer) ring if and only if $R[[x ; \sigma]]$ is a quasi-Baer ring. If $R$ is a ring and $(S, \leq)$ a strictly totally ordered monoid which satisfies the condition that $0 \leq s$ for every $s \in S$, then it is shown in [13] that $R$ is a quasi-Baer ring if and only if the ring $\left[\left[R^{S, \leq}\right]\right]$ of generalised power series over $R$ is a quasi-Baer ring. If $M$ 
is an ordered monoid, then it is proved in [9], Theorem 1, that $R[M]$ is quasi-Baer if and only if $R$ is quasi-Baer. This result has been generalised by G. F. Birkenmeier and J. K. Park in [6], Theorem 1.2, by showing that if $M$ is a u.p.-monoid, then $R[M]$ is quasi-Baer (respectively left principally quasi-Baer) if and only if $R$ is quasi-Baer (respectively left principally quasi-Baer). For skew monoid rings it was proved in [9], Theorem 2, that if $R$ is a ring and $M$ an ordered group acting on $R$, then $R * M$ is a left principally quasi-Baer ring if and only if $R$ is a left $M$-principally quasi-Baer ring. It was also noted in [9], Remark, that if $M$ is an ordered monoid and if there exists a monoid homomorphism $\phi: M \longrightarrow \operatorname{Aut}(R)$ such that $\operatorname{Im}(\phi)$ is a group, then the skew monoid ring $R * M$ is a left principally quasi-Baer ring if and only if $R$ is a left $\operatorname{Im}(\phi)$-principally quasi-Baer ring. Here we have the following result.

THEOREM 5. Let $R$ be a ring, $M$ an ordered monoid and $\phi: M \longrightarrow \operatorname{Aut}(R)$ a monoid homomorphism. Then the following are equivalent:

(1) The skew monoid ring $R * M$ is a left principally quasi-Baer ring.

(2) $R$ is a left $M$-principally quasi-Baer ring.

Proof. (2) $\Longrightarrow(1)$. Suppose that $b_{1} h_{1}+b_{2} h_{2}+\cdots+b_{m} h_{m}$ belongs to $R * M$, and consider the principal left ideal $I=(R * M)\left(b_{1} h_{1}+b_{2} h_{2}+\cdots+b_{m} h_{m}\right)$ of $R * M$. Without loss of generality, we assume that $h_{1}<h_{2}<\cdots<h_{m}$. Let $J$ denote the set of all coefficients of elements of $I$. Then it is easy to see that

$$
J=\sum_{g \in M} R b_{1}^{g}+\sum_{g \in M} R b_{2}^{g}+\cdots+\sum_{g \in M} R b_{m}^{g} .
$$

By point (2), there exists an idempotent $e_{j} \in R$ such that $l_{R}\left(\sum_{g \in M} R b_{j}^{g}\right)=R e_{j}$, $j=1,2, \ldots, m$. Let $e=e_{1} e_{2} \ldots e_{m}$. Then $l_{R}\left(\sum_{g \in M} R b_{1}^{g}+\sum_{g \in M} R b_{2}^{g}+\cdots+\right.$ $\left.\sum_{g \in M} R b_{m}^{g}\right)=\cap_{j=1}^{m} l_{R}\left(\sum_{g \in M} R b_{j}^{g}\right)=R e$. Clearly en $\in l_{R * M}(I)$. Suppose $a_{1} g_{1}+$ $a_{2} g_{2}+\cdots+a_{n} g_{n} \in l_{R * M}(I)$. Then $\quad\left(a_{1} g_{1}+a_{2} g_{2}+\cdots+a_{n} g_{n}\right)(R * M)\left(b_{1} h_{1}+b_{2} h_{2}\right.$ $\left.+\cdots+b_{m} h_{m}\right)=0$. Without loss of generality, we assume that $g_{1}<g_{2}<\cdots<g_{n}$. Suppose that $c_{1}, c_{2}, \ldots, c_{n} \in R$ are such that $a_{i}=c_{i}^{g_{i}}$ for $i=1,2, \ldots, n$. Then, by analogy with the proof of Theorem 2 , we have $c_{i} \in l_{R}\left(\sum_{g \in M} R b_{j}^{g}\right)$ for $i=1,2, \ldots, n$, $j=1,2, \ldots, m$. Thus $c_{i} \in l_{R}(J)$, and so $c_{i}=c_{i} e, i=1,2, \ldots, n$. Now

$$
\left(\sum_{i=1}^{n} a_{i} g_{i}\right)(e \eta)=\sum_{i=1}^{n} a_{i} e^{g_{i}} g_{i}=\sum_{i=1}^{n}\left(c_{i} e\right)^{g_{i}} g_{i}=\sum_{i=1}^{n} c_{i}^{g_{i}} g_{i}=\sum_{i=1}^{n} a_{i} g_{i}
$$

which implies $\sum_{i=1}^{n} a_{i} g_{i} \in(R * M)(e \eta)$. Thus $l_{R * M}(I) \leq(R * M)(e \eta)$. Therefore $l_{R * M}(I)=(R * M)(e \eta)$, and so $R * M$ is a principally quasi-Baer ring.

$(1) \Longrightarrow(2)$. Suppose that the skew monoid ring $R * M$ is a left principally quasiBaer ring. Let $b \in R$. We consider the left annihilator $l_{R}\left(\sum_{g \in M} R b^{g}\right)$. By Hypothesis (1), there exists an idempotent $\alpha \in R * M$ such that $l_{R * M}((R * M)(b \eta))=(R * M) \alpha$. We may write $\alpha=e_{0} \eta+e_{1} g_{1}+\cdots+e_{n} g_{n} \in R * M$, where $e_{i} \in R$ and $\eta, g_{1}, \ldots, g_{n}$ are distinct elements of $M$. Note that the monoid $M$ is cancenllative. For any $r \in R$ and any $g \in M$, from $0=\left(e_{0} \eta+e_{1} g_{1}+\cdots+e_{n} g_{n}\right)(r g)(b \eta)=e_{0} r b^{g} g+e_{1} r^{g_{1}} b^{g_{1} g} g_{1} g+\cdots+$ $e_{n} r^{g_{n}} b^{g_{n} g} g_{n} g$ it follows $e_{0} r b^{g} g=0$, and so $e_{0} r b^{g}=0$. Since $g$ is an arbitrary element of $M$, we have $e_{0}\left(\sum_{g \in M} R b^{g}\right)=0$. Thus $R e_{0} \subseteq l_{R}\left(\sum_{g \in M} R b^{g}\right)$. To prove the converse inclusion, let $a \in l_{R}\left(\sum_{g \in M} R b^{g}\right)$. Then for any $r \in R$ and any $g \in M,(a \eta)(r g)(b \eta)=$ $a r b^{g} g=0$. Thus $(a \eta)(R * M)(b \eta)=0$, and so $(a \eta)=(a \eta) \alpha=(a \eta)\left(e_{0} \eta+e_{1} g_{1}+\cdots+\right.$ $\left.e_{n} g_{n}\right)=a e_{0} \eta+a e_{1} g_{1}+\cdots+a e_{n} g_{n}$. Considering the coefficient of $\eta$ we obtain $a=a e_{0}$. 
Hence $l_{R}\left(\sum_{g \in M} R b^{g}\right) \subseteq R e_{0}$. In particular $e_{0}$ is an idempotent of $R$. Hence $R$ is a left $M$-principally quasi-Baer ring.

Let $M$ be an ordered monoid and $\phi: M \longrightarrow A u t(R)$ a monoid homomorphism. $R$ is called a left $M$-quasi-Baer ring if for any subset $S$ of $R$, the left annihilator of left ideal $\sum_{b \in S} \sum_{g \in M} R b^{g}$ of $R$ is generated by an idempotent of $R$. For the condition that $G$ is a group, left $G$-quasi-Baer rings was considered by Y. Hirano in [9]. Note that by Remark 1(3), left $M$-quasi-Baer rings need not be left quasi-Baer. By analogy with the proof of Theorem 5 we have the following result.

THEOREM 6. Let $R$ be a ring, $M$ an ordered monoid and $\phi: M \longrightarrow A u t(R)$ a monoid homomorphism. Then the following are equivalent:

(1) The skew monoid ring $R * M$ is a quasi-Baer ring.

(2) $R$ is a left $M$-quasi-Baer ring.

Proof. Let $I$ be a left ideal of $R * M$. Denote by $I_{0}$ the set of all coefficients of elements of $I$. Let

$$
J=\sum_{b \in I_{0}} \sum_{g \in M} R b^{g}
$$

If $R$ is left $M$-quasi-Baer, then there exists an idempotent $e \in R$ such that $l_{R}(J)=R e$. Now by analogy with the proof of Theorem 5 we can complete the proof.

COROLlaRY 7. Let $R$ be a ring and $\sigma$ a ring automorphism of $R$. Then

(i) the ring $R[x ; \sigma]$ (respectively $R\left[x, x^{-1} ; \sigma\right]$ ) is left principally quasi-Baer if and only if the left annihilator of $\sum_{i=0}^{\infty} R \sigma^{i}(b)$ (respectively $\left.\sum_{i=-\infty}^{\infty} R \sigma^{i}(b)\right)$ is generated by an idempotent for every $b \in R$;

(ii) the ring $R[x ; \sigma]$ (respectively $R\left[x, x^{-1} ; \sigma\right]$ ) is quasi-Baer if and only if the left annihilator of $\sum_{b \in S} \sum_{i=0}^{\infty} R \sigma^{i}(b)$ (respectively $\left.\sum_{b \in S} \sum_{i=-\infty}^{\infty} R \sigma^{i}(b)\right)$ is generated by an idempotent for any subset $S$ of $R$.

Acknowledgements. The authors wish to express their sincere thanks to the referees for their valuable suggestions and to Professor Li Fang, Zhejiang University, for his help.

\section{REFERENCES}

1. E. P. Armendariz, A note on extensions of Baer and p.p.-rings, J. Austral. Math. Soc. 18 (1974), 470-473.

2. G. F. Birkenmeier, J. Y. Kim and J. K. Park, On quasi-Baer rings, Contemp. Math. 259 (2000), 67-92.

3. G. F. Birkenmeier, J. Y. Kim and J. K. Park, On polynomial extensions of principally quasi-Baer rings, Kyungpook Mathematical J. 40 (2000), 247-254.

4. G. F. Birkenmeier, J. Y. Kim and J. K. Park, Polynomial extensions of Baer and quasiBaer rings, J. Pure Appl. Algebra 159 (2001), 25-42.

5. G. F. Birkenmeier, J. Y. Kim and J. K. Park, Principally quasi-Baer rings, Comm. Algebra 29 (2001), 639-660.

6. G. F. Birkenmeier and J. K. Park, Triangular matrix representations of ring extensions, J. Algebra 265 (2003), 457-477.

7. W. E. Clark, Twisted matrix units semigroup algebras, Duke Math. J. 34 (1967), 417-423.

8. E. Hashemi, The Cohn-Jordan extension and skew monoid rings over a quasi-Baer ring, Commun. Korean Math. Soc. 21 (2006), 1-9. 
9. Y. Hirano, On ordered monoid rings over a quasi-Baer ring, Comm. Algebra 29 (2001), 2089-2095.

10. Y. Hirano, On annihilator ideals of a polynomial ring over a noncommutative ring, J. Pure Appl. Algebra 168 (2002), 45-52.

11. C. Y. Hong, N. K. Kim and T. K. Kwak, Ore extensions of Baer and P.P.-rings, J. Pure Appl. Algebra 151 (2000), 215-226.

12. Z. K. Liu, A note on principally quasi-Baer rings, Comm. Algebra 30 (2002), 3885-3890.

13. Z. K. Liu, Quasi-Baer rings of generalized power series, Chinese Ann. Math. 23 (2002), $579-584$.

14. Z. K. Liu and X. Y. Yang, Triangular matrix representations of skew monoid rings, Math. J. Okayama Univ. 52 (2010), 97-109.

15. Z. K. Liu and R. Y. Zhao, A generalization of PP-rings and p.q.-Baer rings, Glasgow J. Math. 48 (2006), 217-229.

16. B. Stenstrom, Rings of Quotients (Springer-Verlag, New York, 1975).

17. H. Tominaga, On s-unital rings, Math. J. Okayama Univ. 18 (1976), 117-134. 